\title{
ATIVIDADE FÍSICA E GESTAÇÃO: RISCOS E BENEFÍCIOS
}

\author{
Alice de Fátima Negreiros Fumian Ferreira \\ Profissional de Educação Física/Curso de Educação Física (ISECENSA/RJ) \\ alice.fumian@yahoo.com.br
}

Nilo Terra Arêas Neto

Docente do curso de Educação Física (ISECENSA/RJ)/Doutorando em Saúde Coletiva (FIOCRUZ/RJ)

terra.nilo@gmail.com

Anderson Pontes Morales

Docente do curso de Educação Física (ISECENSA/RJ)/Mestre em Ciência da Motricidade Humana (UCB/RJ)

andersonmrl@hotmail.com

\section{Mauricio Rocha Calomeni}

Docente do curso de Educação Física (ISECENSA/RJ)/Doutorando em Saúde Mental (UFRJ/RJ)

mauriciocalomeni@gmail.com

\section{RESUMO}

O objetivo do estudo foi verificar através da pesquisa de campo relatos de experiência sobre a realização de atividades físicas durante a gestação observando seus benefícios para as gestantes e para o desenvolvimento do feto. A pesquisa se caracterizou como ex-post-fact por não tentar manipular a variável dependente, atendo-se a descrever os efeitos do estilo de vida de cada grupo sobre essa variável. Para tanto foram recrutadas em academias e centros de atividades físicas mulheres que durante o período de gravidez praticaram algum tipo de atividade física regular, além disso, também foram recrutadas na comunidade mulheres que não praticaram qualquer tipo de atividade física durante a gravidez. Assim a amostra foi de 16 mulheres sendo que 8 formaram o grupo Gravidez Ativa (GA) e 8 compuseram o grupo Gravidez Sedentária (GS). Foi elaborado especificamente para este estudo um questionário indivídual, e específico para atender as indagações relacionadas à prática de atividade física durante o período gestacional. Concluiu-se conclusão de que mulheres ativas fisicamente na gravidez conseguem manter a rotina do dia-a-dia, tiveram uma recuperação pós-parto excelente. Além disso, foi observado que a inatividade física de um dos grupos de gestantes resultou em um aumento significativo no peso corporal.

Palavras-chave: Atividade Física; Gestação; Educação Física.

\footnotetext{
ABSTRACT

The aim of the study was to verify reports by field research experience on performing physical activities during pregnancy noting its benefits for pregnant women and the developing fetus. The study was regarded as ex-post-fact for not trying to manipulate the dependent variable, sticking to describe the effects of lifestyle of each group on this variable. For both were recruited in gyms and centers for physical activities women during the pregnancy period practiced some form of regular physical activity, in addition, were also recruited women in the community who did not practice any type of physical activity during pregnancy. Thus the sample was 16 women and 8 of the Active Pregnancy 
formed group (GA) and 8 composed the Sedentary Pregnancy group (SG). It was specifically designed for this study an individual questionnaire, and specific to meet the questions related to physical activity during pregnancy. It was concluded conclusion that physically active women in pregnancy can keep the routine day-to-day, had an excellent postpartum recovery. Furthermore, it was observed that physical inactivity of a group of pregnant women resulted in a significant increase in body weight.

Keywords: Physical Activity; pregnancy; Physical Education.

\section{INTRODUÇÃO}

Devido às diversas melhorias na qualidade de vida, e a aversão pela obesidade, cada vez mais se pode encontrar mulheres que no período gestacional, mantêm-se ativas em relação à rotina do dia a dia, frequentando programas de atividade física e alimentando-se de forma correta e saudável. $\mathrm{O}$ exercício regular promove benefícios para a saúde e, praticado durante a gravidez, pode afetar a saúde da mulher para o resto da vida (ARTAL; O'TOOLE, 2003). Assim, diretrizes para a promoção de estilos de vida saudáveis têm sido recomendadas por órgãos envolvidos com a saúde pública, destacando-se a prática de atividade física regular em todo o ciclo vital (BRASIL, 2001). Todavia, essa prática de atividade física só poderá ocorrer quando a mãe apresenta uma gestação sadia, pois, de acordo com a World Health Organization (WHO, 1995), grávidas que mantêm suas atividades diárias sentem-se melhor fisicamente e psicologicamente, conseguindo obter um maior controle sobre seu peso, proporcionando um aumento do peso para o feto. Dessa forma, embora estudos e pesquisas que tratem desse assunto não sejam aprofundados, sabe-se que as grávidas que mantêm um ritmo de atividades, tomando as devidas precauções, têm um período gestacional e pós-parto muito mais tranquilo e saudável (BROWN et al., 2002).

O período gestacional é caracterizado por alterações e adaptações envolvendo os sistemas respiratórios, cardíacos, ósseo e muscular. Para Artal, Clap e Vigil (2000), ocorre ganho de massa corporal localizada na região do abdômen e da pelve, o que faz alterar a postura e o centro de gravidade da gestante, provocando na maioria das mulheres algum desconforto. Já para Chistófalo et al (2003), esse aumento no volume e no peso abdominal acarretar problemas posturais devido ao mecanismo compensatório, com a projeção do corpo para frente. Sendo assim torna-se normal as queixas de dores lombares relatadas pelas gestantes neste período, pois há um o aumento da flexibilidade da coluna e da elasticidade ligamentar. Tais alterações fisiológicas e mecânicas podem ser amenizadas através de programas de atividades físicas orientadas por profissionais da área da Educação Física.

O que se vê na prática é que os benefícios superam os riscos em potencial, se for tomado o devido cuidado ao ministrar o programa de exercícios (SOUZA et al, 2000). Nesse contexto, o American College of Obstetricianand Gynecologistes (ACOG, 1994) desde a década de 90 recomenda a prática de exercícios físicos durante a gestação, podendo esses serem desenvolvidos com ou sem a sustentação de peso, todavia a realização dos exercícios sem impacto facilita a execução. Nesse sentido, sempre deve ser levado em consideração a intensidade, o volume, a sobrecarga e os eixos relacionados à sua prática, uma vez que para uma gestação normal, o exercício de leve a moderado, por pelo menos 30 minutos, não prejudica o desenvolvimento fetal. $\mathrm{E}$ os benefícios da prática regular de exercícios físicos, prescritos corretamente durante a gravidez, ultrapassam, em geral, os riscos potenciais (MCARDLE et al, 2008).

Os benefícios da prática de atividade física durante a gestação são muitos e estão relativamente bem documentados, por meio de metodologias que buscaram quantificar os efeitos que programas de exercícios aplicados durante o período gestacional. Todavia são raros os trabalhos que se voltaram não tanto para o tipo de programa de exercício e/ou intensidade de trabalho feita durante a gestação, mas sim para a percepção das mulheres que se submeteram a exercícios físicos na gestação, sobre os impactos dessa iniciativa na sua saúde e do seu bebê. Então buscando atender a este objetivo, a presente pesquisa buscará responder a seguinte questão: Qual a percepção das mulheres que praticam diferentes tipos de atividades físicas durante a gravidez sobre o impacto na sua saúde e do seu bebê?

Persp. online: biol. \& saúde, Campos dos Goytacazes, 14 (4), 1-8, 2014

seer.perspectivasonline.com.br 


\section{METODOLOGIA}

O presente estudo apresenta características de uma pesquisa de campo de caráter descritivo, pois tentará descrever a influência de uma variável em diferentes grupos. Além disso também tem características de pesquisa ex-post-fact por não tentar manipular a variável dependente, atendo-se a descrever os efeitos do estilo de vida de cada grupo sobre essa variável (THOMAS, NELSON E SILVERMAN, 2012).

A amostra do estudo foi composta por meio do recrutamento de mulheres que durante o período de gravidez tiveram diferentes tipos de experiências relacionadas à atividade física. Para tanto se buscou em academias e centros de atividades físicas mulheres que durante o período de gravidez praticaram algum tipo de atividade física regular, sendo que, para efeito desse estudo entender-se-á como praticante de atividade física, pessoas que dedicam pelo menos trinta minutos do seu tempo na realização de exercícios físicos ao menos três vezes por semana. Além disso, também serão recrutadas na comunidade mulheres que não praticaram qualquer tipo de atividade física durante a gravidez.

Para se manter o maior homogeneidade da amostra todas as mulheres, deveriam ser economicamente ativas e ter feito o pré-natal regularmente. Através disso foi reunida uma amostra de 16 mulheres $(n=16)$, sendo que dessas, $8(n=8)$ formaram o grupo Gravidez Ativa (GA) e as outras 8 $(\mathrm{n}=8)$ compuseram o grupo Gravidez Sedentária (GS).

Todas as mulheres foram voluntárias e concordaram com a participação no estudo atraevés de assinatura de Termo de Consentimento Livre e Esclarecido. A pesquisa foi avaliada e aprovada pelo comitê de ética dos Institutos Superiores de Ensino do CENSA com parecer n ${ }^{\circ} 760.847$

Foram incluídas nesse grupo mulheres economicamente ativas, que tiveram filhos a menos de 10 anos, que realizaram normalmente todo o pré-natal e que tenham ou não realizado exercícios físicos durante este período. Sendo inseridas nos respectivos grupos a seguir, de acordo com este dado sobre a prática de atividades físicas:

\subsection{Grupo Gravidez Ativa (GA)}

Mulheres que durante agravidez tenham praticado atividades físicas ao menos três vezes por semana com duração de 30 minutos.

\subsection{Grupo Gravidez (GS)}

Mulheres que durante agravidez não tenham praticado atividades físicas regularmante.

Foram excluídas mulheres que não eram economicamente ativas, que tiveram filhos a mais de 10 anos, que não realizaram normalmente todo o pré-natal.

Foi elaborado especificamente para este estudo um questionário indivídual, e específico para atender as indagações relacionadas à prática de atividade física durante o período gestacional. $\mathrm{O}$ questionário aplicado foi composto por 15 (quinze) perguntas divididas em blocos. O bloco um aborda sobre as características pessoais das entrevistadas e aspectos voltadospara o período gestacional. $\mathrm{O}$ bloco dois refere-se à prática de regular atividade física. E por fim, o bloco três é voltado para a percepção da própria mãe, em relação aos benefícios de ter se mantido ativa durante a gravidez.

$\mathrm{O}$ referido questionário foi aplicado de forma individual, e para tanto a pesquisadora foi ao encontro dos avaliados em dia e hora previamente definidos.

Os dados provenientes da aplicação do questionario desenovolvido para o estudo foram tratados descritivamente por meio da média e do desvio padrão das idades em que as mulheres engravidaram e de quantos quilos engordaram. Para as outras respostas dadas a cada uma das perguntas feitas foi utilizada a moda para caracterizar cada grupo.

Para analise individual das questões mais relevantes para atender os objetivos do estudo foi utilizado os valores percentuais relativos a cada resposta dada, e na análise inferencial foi utilizado o Teste Exato de Fisher, sendo fixado o nível de significância em $\mathrm{p}<0.05$.

\section{RESULTADOS e DISCUSSÃO}

Findada a aplicação dos questionários nas mulheres participantes da pesquisa, agrupadas em 
praticantes de atividade física (AF) durante a gravidez, e, não praticantes de AF durante a gravidez, os dados foram compilados na tabela 1 para melhor apresentação e interpretação dos mesmos.

Tabela 1. Apresentação dos dados provenientes dos questionários aplicados nas mulheres que constituíram os grupos da pesquisa.

\begin{tabular}{|c|c|c|}
\hline & $\begin{array}{l}\text { Praticantes de AF } \\
\qquad(\mathrm{n}=8)\end{array}$ & $\begin{array}{c}\text { Não Praticantes de AF } \\
\qquad(\mathrm{n}=8)\end{array}$ \\
\hline \multicolumn{3}{|c|}{ SOBRE A GRAVIDEZ } \\
\hline 2. Idade em que engravidou? & $28 \pm 5,4$ & $28 \pm 4,6$ \\
\hline 3. Teve acompanhamento médico? & Sim & Sim \\
\hline 4. Como foi a gestação? & Normal & Normal \\
\hline $\begin{array}{l}\text { 5. Quantos quilogramas você engordou } \\
\text { durante este período? }\end{array}$ & $12,2 \pm 5,1$ & $16,9 \pm 7,5$ \\
\hline 6. Como foi o parto? & Cesariana & Cesariana \\
\hline 7. Peso do bebê ao nascer? & Normal & Normal \\
\hline $\begin{array}{l}\text { 8. Com que frequência semanal praticava } \\
\text { atividade física antes da gravidez? }\end{array}$ & 4 & 2 \\
\hline \multicolumn{3}{|c|}{ SOBRE A ATIVIDADE FÍSICA } \\
\hline $\begin{array}{l}\text { 1. Tipo de atividade que praticava antes da } \\
\text { gravidez? }\end{array}$ & $\begin{array}{l}\text { Musculação e } \\
\text { aeróbio }\end{array}$ & Aeróbio \\
\hline $\begin{array}{l}\text { 2. Houve Orientação de um profissional em } \\
\text { Educação Física? }\end{array}$ & Sim & Sim \\
\hline $\begin{array}{l}\text { 3. Ao engravidar manteve a rotina do dia-a- } \\
\text { dia? }\end{array}$ & Sim & Não \\
\hline 4. O que mudou? & Nada mudou & $\begin{array}{c}\text { Ansiedade } \\
\text { /Insegurança/Medo }\end{array}$ \\
\hline \multicolumn{3}{|c|}{ PERCEPÇÃO DA MÃE } \\
\hline 1. Como foi a recuperação pós-parto? & Excelente & Normal/Lenta \\
\hline $\begin{array}{l}\text { 2. Na sua percepção o fato de ter se mantido } \\
\text { ativa durante a gestação trouxe algum } \\
\text { benefício para o desenvolvimento do feto? }\end{array}$ & Sim & --- \\
\hline
\end{tabular}

A análise dos dados da tabela 1 mostra que com relação à idade na qual engravidaram os grupos foram homogêneos, ou seja, em média as mulheres engravidaram aos 28 anos. Todas tiveram gestação normal, receberam acompanhamento médico durante a gravidez, e, o tipo de parto mais relatado em ambos os grupos foi cesariana. Porém uma das mulheres entrevistadas pertencente do grupo de mulheres ativas durante a gestação, relatou que passou por duas gestações, sendo que na primeira praticou em seu dia-a-dia musculação, e seu parto foi normal, já na segunda gestação, após 2 anos, não praticou atividada física regularmente, e seu parto foi cesariana. $\mathrm{O}$ interessante referente a este fato é que esta mulher gestante relatou com base na sua autopercepção que o fato de ter realizado musculação na primeira gestação ajudou muito para para que houvesse a possibilidade de realizar o parto normal, o que não foi observado na segunda gestação.

Outro fato é que a grande maioria dos bebês nasceu com peso normal independentemente do grupo no qual as mulheres estavam inseridas. Tais informações são relevantes uma vez que ressaltam a homogeneidade dos grupos e reforçam a ideia que as possíveis diferenças encontradas entre os grupos se deram devido à prática da atividade física durante a gravidez.

Uma das primeiras diferenças perceptíveis entre os grupos é que as mulheres que não praticaram atividades físicas durante a gestação engordaram em média 4,7 quilos a mais que as mulheres que praticaram atividades físicas durante a gravidez, e esse sobrepeso pode estar relacionado 
a certos riscos tanto para mãe quanto para o bebê. O American College of Obstetricians and Gynecologists (ACOG, 1994) reconhece que o controle do ganho de peso na gestação e no pós-parto, sofrem influencia através do suprimento nutricional adequado estando relacionado com a prática regular de atividade física relizada neste período. Segundo Sebire (2001) as gestantes obesas também apresentam maior probabilidade de terem infecções urinárias e do trato genital inferior aumentando o risco de hemorragia maciça pós-parto e infecção puerperal.

A literatura de Anderson et al (2005) e Ray et al (2004) nos mostra que a taxa de malformações fetais é maior em mulheres obesas do que naquelas com peso normal. $\mathrm{O}$ excesso de tecido adiposo materno parece ainda ser capaz de comprometer a programação metabólica fetal, predispondo os filhos de mulheres obesas a serem futuros obesos e diabéticos (WHITAKER, $2004 \mathrm{e}$ SIEGA-RIZ e LARAIA, 2006), perpetuando o ciclo da obesidade. Além disso, a incidência de Diabetes Melito gestacional (DMG) em gestantes obesas é três vezes maior que na população geral.

O ganho de peso ideal na gestação é baseado nas recomendações do Institute of Medicine (IOM-2009) e leva em consideração o IMC pré-concepcional da paciente. Mulheres que apresentam IMC 18,5 (Baixo peso), podem engordar de 12,5Kg à 18Kg; IMC 18,5 à 24,9 (Peso adequado), podendo engordar de 12 à $18 \mathrm{Kg}$; IMC 25 à 29,9 (Sobrepeso) podem engordar de $7 \mathrm{Kg}$ a $11 \mathrm{Kg}$ e por fim as gestantes que apresentam IMC 30, são classificadas em obesas, portanto são recomendadas apenas um ganho de peso variando de $5 \mathrm{~kg}$ a $9 \mathrm{~kg}$.

Provavelmente esse sobrepeso observado nas mulheres do grupo que não praticaram $\mathrm{AF}$ durante a gravidez está relacionado a menor frequência semanal que estas se exercitavam antes de engravidar como ilustrado na figura 1.

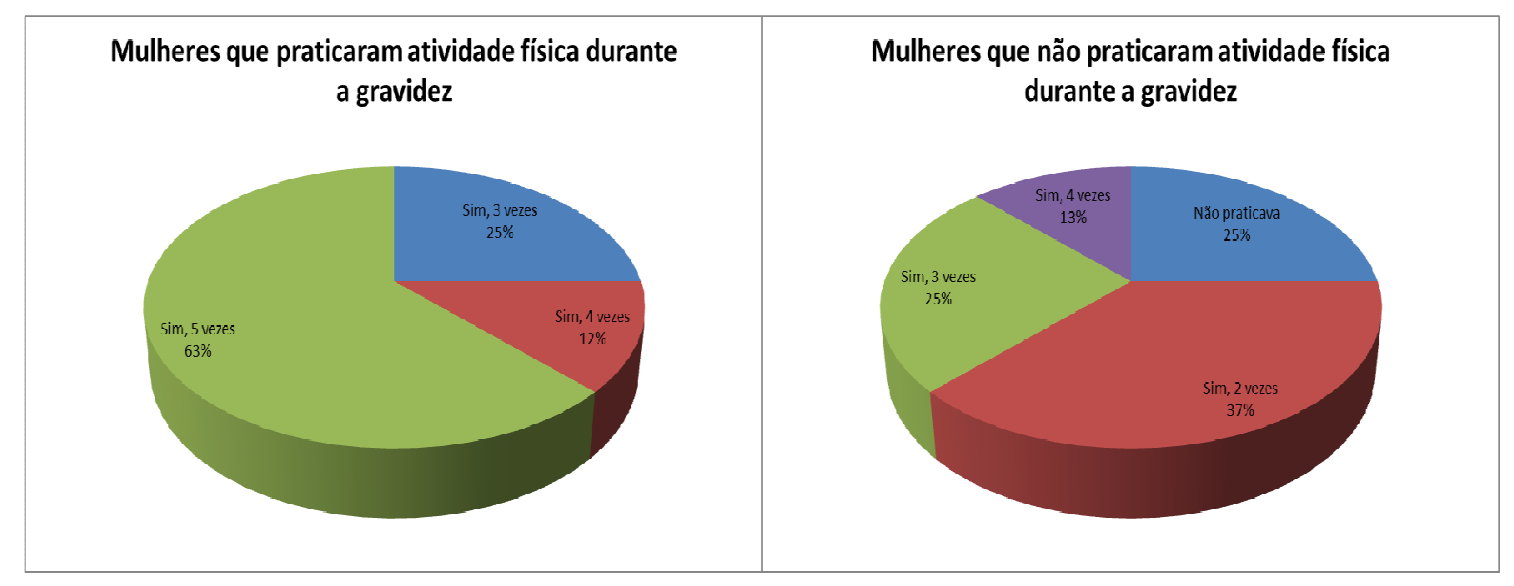

Figura 1. Percentis de respostas dadas à pergunta "Praticava atividade física antes de engravidar? Com que frequência semanal?", nos dois grupos que compuseram a pesquisa.

Analisando a figura 2, fica claro estatisticamente através da inferência feita pelo Teste Exato de Fisher $(\mathrm{p}<0,05)$ que a prática de $\mathrm{AF}$ durante a gravidez proporcionou as mulheres manterem sua rotina normal durante esse período. Os benefícios relacionados a prática de Atividade Física durante a gestação podem ser divididos em dois tipos: biológicos e psicosociais (MATSUDO et al, 2000). Segundo Santarém (2000) dentre os benefícios biológicos, podemos destacar a independência funcional, ou seja, a capacidade de realizar as atividades desejadas do dia-dia sem que haja riscos para a integridade física do organismo. E os benefícios psicosociais englobam alterações de comportamento e sensações, exercendo influência sobre as atitudes tomadas pelas mulheres durante a gestação.

No grupo de mulheres não praticantes de atividade física durante a gestação não foi obsevado a manutenção da rotina diária. Durante a pesquisa o grupo relatou insegurança, ansiedade e medo de prejudicar o bebê como motivo para não praticarem atividades físicas durante a gravidez. 


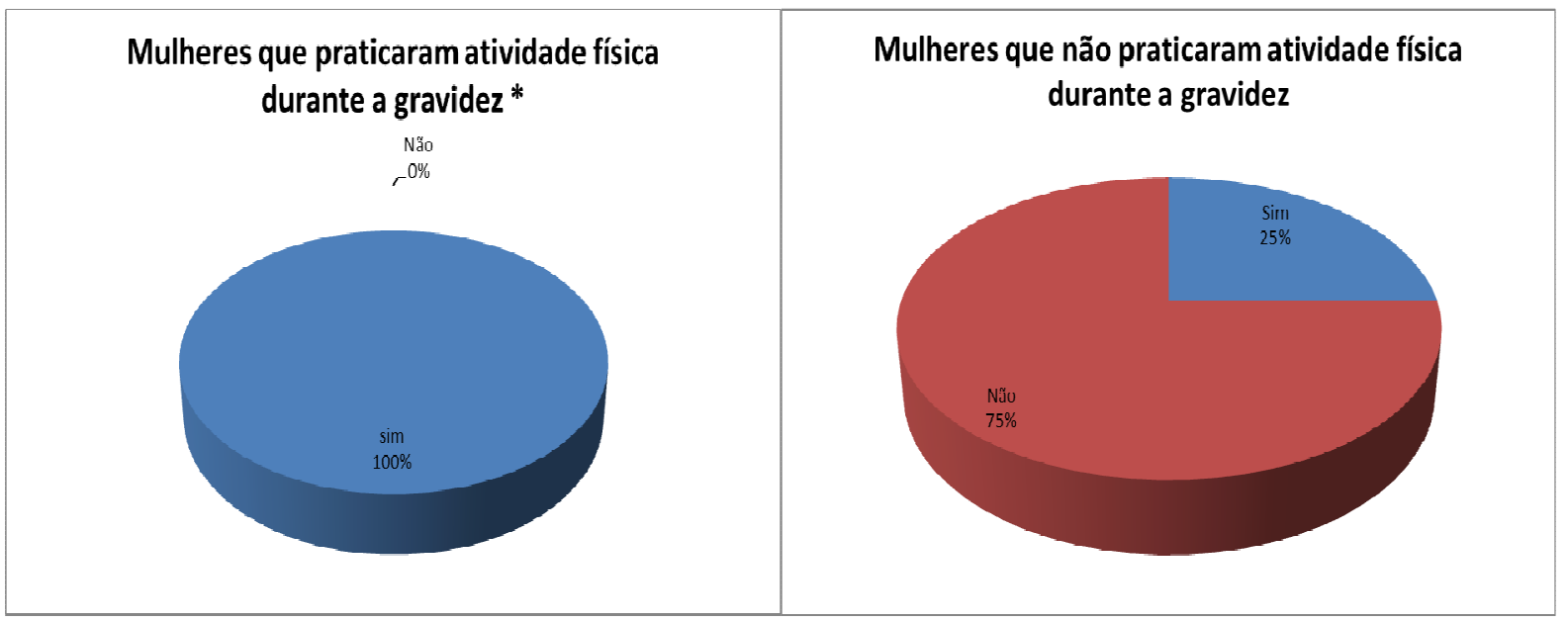

Figura 2. Percentis de respostas dadas à pergunta “Ao engravidar manteve a rotina do dia-a-dia?", nos dois grupos que compuseram a pesquisa. *Valor de $\mathrm{p}=0,007$ no Teste Exato de Fisher.

Outro fato interessante, e facilmente observável na figura 3 apesar da falta de significância estatística, é que as mulheres do grupo que praticou AF durante a gravidez relataram em sua grande maioria (75\%), que a recuperação pós-parto foi excelente. De acordo Artal, Clapp e Vigil (2000) pesquisas demonstraram que a participação regular de grávidas em um programa de atividades físicas pode melhorar o condicionamento fisiológico das mães, restringir o ganho de peso sem comprometer o desenvolvimento do feto e ainda facilitar a recuperação pós-parto, enquanto que no grupo formado por mulheres que não praticaram atividade física durante a gravidez se teve relatos que variaram de recuperação lenta ( 2 relatos) até excelente, sendo que os mais mencionados foram excelente e normal com 3 ocorrências cada.

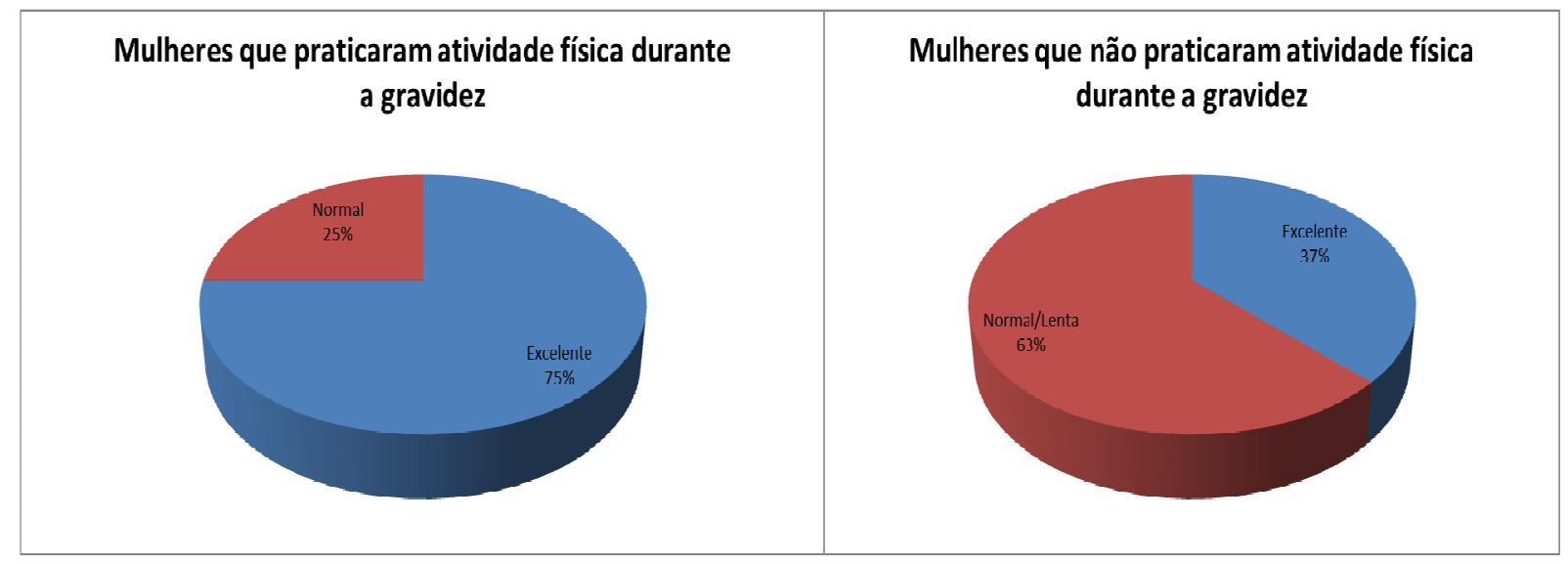

Figura 3. Percentis de respostas dadas à pergunta "Como foi a recuperação pós-parto?", nos dois grupos que compuseram a pesquisa. Valor de $\mathrm{p}=0,31$ no Teste Exato de Fisher.

Além disto, importate relatar que o grupo que praticou $\mathrm{AF}$ teve a percepção de que ter se mantido ativas durante a gestação trouxe benefícios para formação do feto, enquanto que as mulheres do grupo que não praticou AF não puderam ter tal percepção.

\section{CONCLUSÕES}

Após a realização desta pesquisa se pode observar que mulheres ativas fisicamente na gravidez conseguem manter a rotina do dia-a-dia, além disso, a percepção da recuperação pósparto, nas mães que se mantiveram ativas foi exelente, não sendo relatado prejuízo para a formação do feto, e, pelo contrário, as mães perceberem que o fato de terem se mantido ativas resultou em benefícios. Além disso, foi observado que a inatividade física de um dos grupos de gestantes resultou em um aumento no peso corporal das mesmas. 
Todavia, é muito importante que sejam tomadas precauções com relação ao tipo de atividade a ser praticada, sua intensidade, carga, local de realização e posição adequada no qual a gestante deve permanecer durante a prática, para que não prejudique a saúde de ambos. Este período não deve ser marcado por um momento em que a gestante deva sair do sedentarismo, mas sim dar continuidade a prática de exercícios físicos, lembrando que é presciso que esteja tudo bem com a gestante e com o bebê para que seja desenvolvido de quaquer programa de atividade física.

\section{REFERÊNCIAS}

ARTAL, R.; O'TOOLE, M. Guidelines of the American College of Obstetricians and Gynecologists for exercise during pregnancy and postpartum period. British Journal of Sports Medicine, St. Louis, USA, v. 37, n. 1, p. 6-12, feb. 2003 . Disponívelem: < http://www.luzimarteixeira.com.br> Acesso 18 de março de 2014.

BRASIL. Ministério da Saúde. Áreas técnicas. Projetos de promoção a saúde. 2001. Disponível em: < http://www.saude.gov.br >Acesso em: 18 de março de 2014

WHO. World Health Organization. Physical status: the use and interpretation of anthropometry. Report of a WHO Expert Committee. Geneve, 1995. WHO-Technical Report Series no 854.

BROWN W. et al, The benefits and risks of exercise during pregnancy. Medicine Science Sports and Exercise, v. 5, p. 11-29, 2002. Disponível em: < http://www. efdesportes.com/revistadigital > Acesso em: 28 de março de 2014.

ARTAL, Raul., CLAPP, James F., VIGIL, Daniel V. Exercícios durante a gravidez: recomendações do American College of Sports Medicine, 2000 Disponível em: <http://www.efartigos.hpg.com.br/ otemas/artigo53.html> . Acesso: 16 jul. 2014.

CHRISTÓFAlO, C.; MARTINS, A.J. \& TUMELERO, S. A prática de exercício físico durante o período de gestação Artal, Clapp. Revista Digital: Buenos Aires, 2003. Disponível em: $<$ http://www.efdeportes.com> Acesso 03 de novembro 2014.

SOUZA, E. L. B. L. Fisioterapia aplicada à obstetrícia e aspectos de neonatologia:uma revisão multidisciplinar. 2. ed. Belo Horizonte: Health, 2000.

ACOG (American College of Obstetricians and Gynecologists). Exercise during pregnancy and the postpartum period. Washington (DC): O College: 1994.

SEBIRE, N.J. Maternal obesity and pregnancy outcome: a study of 287,213 pregnancies in London.International Journal of Obesity and Related Metabolic Disorders; 25: 8, 1175-1182, 2001.

MCARDLE, W.D.; KATCH, F.I.; KATCH, V.L. Fisiologia do exercício: Energia, nutrição e desempenho humano. $6^{\text {a }}$ ed. Rio de Janeiro: Guanabara Koogan, 2008.

THOMAS, J.; NELSON, J.; SILVERMAN, S. Métodos de pesquisa em atividade física.Porto Alegre: Artmed, 2012. $6^{\circ}$ ed.

MATSUDO, V.K.R; MATSUDO, S.M.M \& TEDESCO, J,J. Atividade Física e Esportiva na Gravidez. A gravida. São Paulo: Atheneu, p. 53-81, 2000. Disponível em: < http://www disponível em: http://files.adrianobelem.webnode.com.br >Acesso em 13 de março 2014

ANDERSON, J.L.,WALLER, D.K.,CANFILD, M.A., SHAW, G.M., WATKINS, M.L., WEELER, M.M. Maternal obesity, gestational diabetes, and central nervous systembirth defects. Epidemiology. 2005;16(1):87-92. 
RAY, J.G., VERMEULEN, M.J., MEIER, C., WYATT, P.R. Risk of congenital anomalies detected during antenatal serum screening in women with pregestational diabetes. QJM. 2004;97(10):651-3.

WHITAKER, R.C. Predicting preschooler obesity at birth: the role of maternal obesity in early pregnancy. Pediatrics. 2004;114(1):e29-36.

SIEGA-RIZ, A.M., LARAIA, B. The implications of maternal overweight and obesity on the course of pregnancy and birth outcomes. Matern Child Health J. 2006;10(5 Suppl):S153-6.

SANTARÉM, José Maria. Hipertrofia muscular: aptidão física, saúde e qualidade de vida. Acadêmico de Educação Física pela UFRN Editor: Allan José Costa - Revista Virtual EFArtigos. Disponível em: <http://www.saudetotal.com/saude/hptrofiq.htm. >Acesso: 16 jul. 2014. 